\title{
Early and late mortality after malaria in young children in Papua, Indonesia
}

\author{
Dewi Patriani ${ }^{1}$, Eggi Arguni ${ }^{1}$, Enny Kenangalem², ${ }^{2,}$ Saber Dini ${ }^{4}$, Paulus Sugiarto ${ }^{5}$, Afdhal Hasanuddin ${ }^{5}$, \\ Daniel Adrian Lampah², Nicholas M. Douglas ${ }^{6}$, Nicholas M. Anstey ${ }^{6,7}$, Julie Anne Simpson ${ }^{5}$, Ric N. Price ${ }^{6,8,9}$ and \\ Jeanne Rini Poespoprodjo ${ }^{1,2,3^{*}}$
}

\begin{abstract}
Background: In southern Papua, Indonesia, malaria is highly prevalent in young children and is a significant cause of morbidity and early mortality. The association between malaria and delayed mortality is unknown.

Methods: Routinely-collected hospital surveillance data from southern Papua, Indonesia, were used to assess the risk of recurrent malaria and mortality within 12 months of an initial presentation with malaria in all children younger than 5 years old attending the local hospital. Analysis was primarily by Kaplan Meier and Cox regression methods.

Results: In total 15,716 children presenting with malaria between April 2004 and December 2013 were included in the analysis; 6184 (39.3\%) with Plasmodium falciparum, 7499 (47.7\%) with P. vivax, 203 (1.3\%) with P. malariae, 3 with $P$. ovale and 1827 (11.6\%) with mixed infections. Within 1 year, 48.4\% (7620/15,716) of children represented a total of 16, 957 times with malaria (range 1 to 11 episodes), with the incidence of malaria being greater in patients initially presenting with $P$. vivax infection (1334 [95\%Cl 1307-1361] per 1000 patient years) compared to those with $P$. falciparum infection (920 [896-944]). In total 266 (1.7\%) children died within 1 year of their initial presentation, 129 (48.5\%) within 30 days and 137 (51.5\%) between 31 and 365 days. There was no significant difference in the mortality risk in patients infected with P. vivax versus P. falciparum either before 30 days (Hazard Ratio (HR) 1.02 [0.69,1.49]) or between 31 and 365 days ( $\mathrm{HR}=1.30[0.90,1.88]$ ). Children who died had a greater incidence of malaria, 2280 [95\%Cl 1946-2671] per 1000 patient years preceding their death, compared to 1141 [95\%Cl 1124-1158] per 1000 patient years in those surviving.

Conclusions: Children under- 5 years old with $P$. vivax malaria, are at significant risk of multiple representations with malaria and of dying within 1 year of their initial presentation. Preventing recurrent malaria must be a public health priority in this vulnerable population.
\end{abstract}

Keywords: P. falciparum, P. vivax, Infants and children under-fives, Mortality

\section{Background}

Children under 5 years of age, living in malaria endemic areas are particularly vulnerable to malaria, its severe manifestations and malaria attributable mortality [1]. In Papua Province, eastern Indonesia, recurrent episodes of malaria occur frequently in early life and are associated with significant morbidity and mortality [2, 3]. The severe

\footnotetext{
* Correspondence: didot2266@yahoo.com

'Department of Child Health, Faculty of Medicine, Public Health and Nursing, Universitas Gadjah Mada, Jl. Kesehatan no.1, Sekip, Yogyakarta 55284, Indonesia

${ }^{2}$ Timika Malaria Research Programme, Papuan Health and Community Development Foundation, JI. SP2-SP5, RSMM Area, Timika, Papua 99910 Indonesia

Full list of author information is available at the end of the article
}

manifestations of Plasmodium falciparum malaria are well recognized, and there is now good evidence of early morbidity and mortality associated with Plasmodium vivax infection in some endemic regions [3, 4]. Pauci-immune young children are at risk of recurrent episodes of malaria, and in Asia this is particularly apparent following $P$. vivax infection.

Malaria control and elimination efforts are more challenging for $P$. vivax than $P$. falciparum and consequently in many co-endemic settings, there has been a rise in the relative proportion of malaria attributable to $P$. vivax. Furthermore without radical cure of the dormant liver stages of $P$. vivax, recurrent parasitaemia occurs every 3-4 weeks in equatorial regions [5] resulting in a cumulative risk of severe anaemia [6].

(c) The Author(s). 2019 Open Access This article is distributed under the terms of the Creative Commons Attribution 4.0 International License (http://creativecommons.org/licenses/by/4.0/), which permits unrestricted use, distribution, and 
The aetiology of $P$. vivax mortality remains controversial, however malnutrition, severe anaemia and bacterial sepsis are potentially important contributing factors $[7,8]$. The long-term impact of recurrent malaria in young children is poorly described. This study aimed to quantify and compare the early and late mortality of children less than 5 years of age following presentation with $P$. vivax and/or $P$. falciparum infection in Papua, Indonesia.

\section{Methods}

\section{Study site}

Timika is located in southern Papua, the most easterly province of Indonesia. A local census in 2012, recorded the population of Mimika district to be 202,350 with almost $90 \%$ of the population located in Timika town and surrounding villages serviced by the Mitra Masyarakat hospital (RSMM). The area has fragmented forest with a climate that varies little throughout the year (unpublished data). Malaria is restricted to lowland areas where transmission is unstable. The annual incidence of clinical malaria during the study period declined from 889 per 1000 population in 2004 to 522 per 1000 in 2013 and over the same period the proportion of infections due to $P$. vivax rose from 44 to $53 \%$ [9]. Local $P$. vivax strains have a typical relapse interval of 3-4 weeks and are highly resistant to chloroquine $[10,11]$. Approximately half of the population own insecticide treated bednets [12].

Rumah Sakit Mitra Masyarakat (RSMM) is the largest health care facility in Timika. During the study period, RSMM treated between 40 and $60 \%$ of patients diagnosed with malaria in the district [12]. A second hospital opened in December 2008, but did not begin seeing substantial numbers of malaria patients until 2010. From 2010 to 2013 , RSMM received an estimated $80 \%$ of malaria patients attending either of the two hospitals [9]. RSMM offers free medical care for most patients of lowland or highland Papuan ethnicity, who make up 93\% of attendees, and as such is the preferred source of medical care for inpatient, outpatient and antenatal encounters [12]. A community household survey of treatment seeking behavior in 2005 , estimated that more than $80 \%$ of children under 5 who had died in the preceding year had done so at the RSMM hospital [13].

\section{Study population}

The local population is made up of ethnic groups that can be divided broadly into highland and lowland Papuans, with a migrant non-Papuan population originating mostly from low or non-malaria endemic regions. Diarrhea, lower respiratory tract infections and malaria are the commonest causes of morbidity and mortality in young children. Excluding neonatal deaths, the underfive mortality rate in babies born at RSMM between 2004 and 2013 was 17 per 1000 live births (unpublished data). Insecticide treated bednets have been widely distributed since 2004 and are used by approximately half of the population [12].

\section{Study design}

Data were gathered prospectively from routine documentation of patients presenting to the RSMM hospital. No patient data were gathered on events occurring at other healthcare facilities or outlets. Between April 2004 and December 2013 patients presenting to RSMM had clinical, laboratory and pharmacy data recorded in a Q-Pro ${ }^{\mathrm{Tm}}$ database and could be identified by a unique hospital record number. Data recorded included date of presentation and discharge, age, sex, ethnicity, clinical diagnoses (ICD 10) assigned by the attending physician, full blood count parameters and prescription details. The analysis included all children aged 5 years or under at the time of their first presentation (either as an inpatient or outpatient) to hospital with malaria. All subsequent visits to hospital were recorded, including re-presentation with and without malaria. Babies in the first month of life were excluded to avoid confounding from perinatal mortality and congenital malaria $[14,15]$. Hospital protocol dictates that any patient presenting with fever, a history of fever or severe illness should be checked for malaria by microscopy using Giemsa stained thick blood smears. Review and quality assurance demonstrated that this protocol was well adhered to with microscopy accuracy of $90 \%$ compared to expert review [3]. Prior to 2006, the first line local treatment for uncomplicated malaria was chloroquine plus sulphadoxinepyrimethamine for $P$. falciparum and chloroquine for $P$. vivax, although in the RSMM hospital most patients were treated with a 7 day oral regimen of quinine. Patients with $P$. vivax were also offered a 14 day course of low-dose primaquine $(3.5 \mathrm{mg} / \mathrm{kg}$ total dose). In view of the poor efficacy of these regimens, the standard protocol was changed in March 2006 to dihydroartemisinin-piperaquine (DHP) for all Plasmodium species plus 14 days primaquine in those with $P$. vivax infection [16]. At the same time the recommended treatment for severe malaria was changed from intravenous quinine to intravenous artesunate [17].

\section{Definitions}

Infants were defined as children aged less than 1 year old and young children as those aged 1 to less than 5 years old. Children under 5 years old include both infants and young children. A diagnosis of malaria was only made following confirmation of parasitaemia by blood film microscopy. Mortality was defined as death during admission due to any cause and categorized as early (within 30 days) or late (from 31 to 365 days of the first hospital presentation with malaria). Severe anaemia was defined as a haemoglobin concentration below $7 \mathrm{~g} /$ dl. Malnutrition was assessed by the attending physician 
and defined as weight for age or height/length for weight $\mathrm{z}$ scores below -3SD of standard reference at that time $[18,19]$.

\section{Statistical analysis}

Data were analysed using SPSS version 20.0 for windows software (IBM SPSS Statistics) and Stata version 14.2 (StataCorp, College Station, TX, US). Clinical, laboratory and pharmacy data were concatenated such that multiple presentations with malaria within 14 days of the initial presentation were grouped as a single "episode", the rationale being that recurrent parasitemia due to reinfection or relapse is highly unlikely within this period [20]. KaplanMeier survival curves for the risk of re-presentation to hospital with malaria and the risk of any hospital presentation terminating in death were plotted stratified by the baseline characteristics sex, ethnicity (non-Papuan, Highland Papuan, Lowland Papuan), age group, year of initial presentation, presence or absence of Plasmodium co-infection and initial admission status (outpatient or inpatient). Cox proportional hazards regression models were used to identify risk factors for recurrent malaria and early and late mortality. The proportional hazards assumption was assessed by comparing visually the log (cumulative hazard) by time of follow-up curves for each covariable category and subsequently by fitting and comparing models with and without time of follow-up interaction terms. Univariable analyses were performed for each of the following variables: Plasmodium species, sex, self-reported ethnicity (non-Papuan, Highland Papuan, Lowland Papuan), age group $(<1$ year and 1 to $<5$ years), inpatient status, presence of severe anaemia $(\mathrm{Hb}<7 \mathrm{~g} / \mathrm{dl}$ or $<5 \mathrm{~g} / \mathrm{dl})$ and malnutrition. All of these factors were included in multivariable models. In view of the change of treatment policy, antimalarial treatment regimens were collinear with the year of the study, thus all multivariable models were stratified by year. For late mortality, we also performed subgroup analyses by whether the child was an outpatient for the initial malaria episode or admitted to hospital for management of the infection.

In survival analysis and the calculation of incidence rates, the period of observation was defined as the time from first presentation with malaria until 12 months, the time of recorded death or the end of the study period (31st December 2013), whichever occurred earliest. Population attributable fractions (PAF) were calculated using STATA 14.2 to calculate PAFs as described previously [21].

\section{Ethical approval}

Ethical approval for this study was obtained from the Medical and Health Research Ethics Committee, Faculty of Medicine, Gadjah Mada University, Yogyakarta, Indonesia (KE/FK/544/EC), Menzies School of Health Research, Darwin, Australia (HREC 10.1397).

\section{Results}

Between April 2004 and December 2013, 1,054,674 clinical presentations to RSMM were recorded, of which 196 , $380(18.6 \%)$ episodes in 68,530 patients included a diagnosis of malaria. A total of 15,716 children were aged between 1 month and 5 years old at their first presentation to hospital with malaria, of whom 6184 (39.3\%) had P. falciparum malaria, 7499 (47.7\%) had P. vivax, 203 (1.3\%) had P. malariae, $3(0.02 \%)$ had P. ovale and $1827(11.6 \%)$ had mixed infections. Overall 12,192 (77.6\%) were treated as outpatients and 3524 (22.4\%) required admission for inpatient care. The study profile is presented in Fig. 1 and the baseline characteristics in Table 1.

At the initial presentation, $25.2 \%(1148 / 4561)$ of infants and $21.3 \%(2376 / 11,155)$ of young children required hospitalization for treatment of their malaria. Children presenting with $P$. falciparum infection were more likely to require inpatient treatment compared to those with $P$. vivax (Odds Ratio $(\mathrm{OR})=2.31 \quad[95 \% \mathrm{CI}$ 2.12-2.51], $p<0.001)$. Haemoglobin concentration was recorded during the hospital presentation in 8687 (55.3\%) of children and the mean haemoglobin was 8.12 $\mathrm{g} / \mathrm{dl}[\mathrm{SD}=2.4]$. Severe anaemia was present in $30.8 \%$ $(2674 / 8687)$ of cases $(\mathrm{Hb}<7 \mathrm{~g} / \mathrm{dl})$.

The first episode of malaria could be matched with pharmacy records in $92.1 \%(14,472 / 15,716)$ of cases. Prior to March 2006, 69.2\% (2341/3385) received oral treatment with quinine or chloroquine and 23.2\% (784/ $3385)$ were treated with intravenous quinine with or without an oral regimen. Following the change in policy in March 2006, 96.6\% (10,708/11,087) received oral treatment with an artemisinin-based combination therapy (ACT) and $19.3 \%(2136 / 11,087)$ were treated with intravenous artesunate with or without an oral regimen. In total $52.6 \%(7693 / 14,627)$ of children were also prescribed primaquine (PQ), of whom $23.5 \%$ (1810) were prescribed a single dose of $\mathrm{PQ}$ and $70.3 \%$ (5405) were prescribed 14 day regimens; for $478(6.2 \%)$ children, the dose of PQ could not be defined.

\section{Risk factors for recurrent malaria}

Within 12 months of their first presentation 7620 (48.4\%) of children had at least one re-presentation with malaria (total number of re-presentations $=16,958$, range 1 to 11); Fig. 2a. In infants, 63.9\% (4144/6481) of all re-presentations with malaria were due to $P$. vivax, but this fell to $50.4 \%(5280 / 10,477)$ in young children; $p<0.0001$. The incidence of malaria within 1 year was 1334 [95\%CI 1307-1361] per 1000 person-years (py) in patients initially presenting with $P$. vivax compared to 920 [896-944] per 1000 py for those initially presenting with P. falciparum, Rate ratio 1.45 [95\%CI 1.40-1.50], $p<0.001$; Table 2 . 


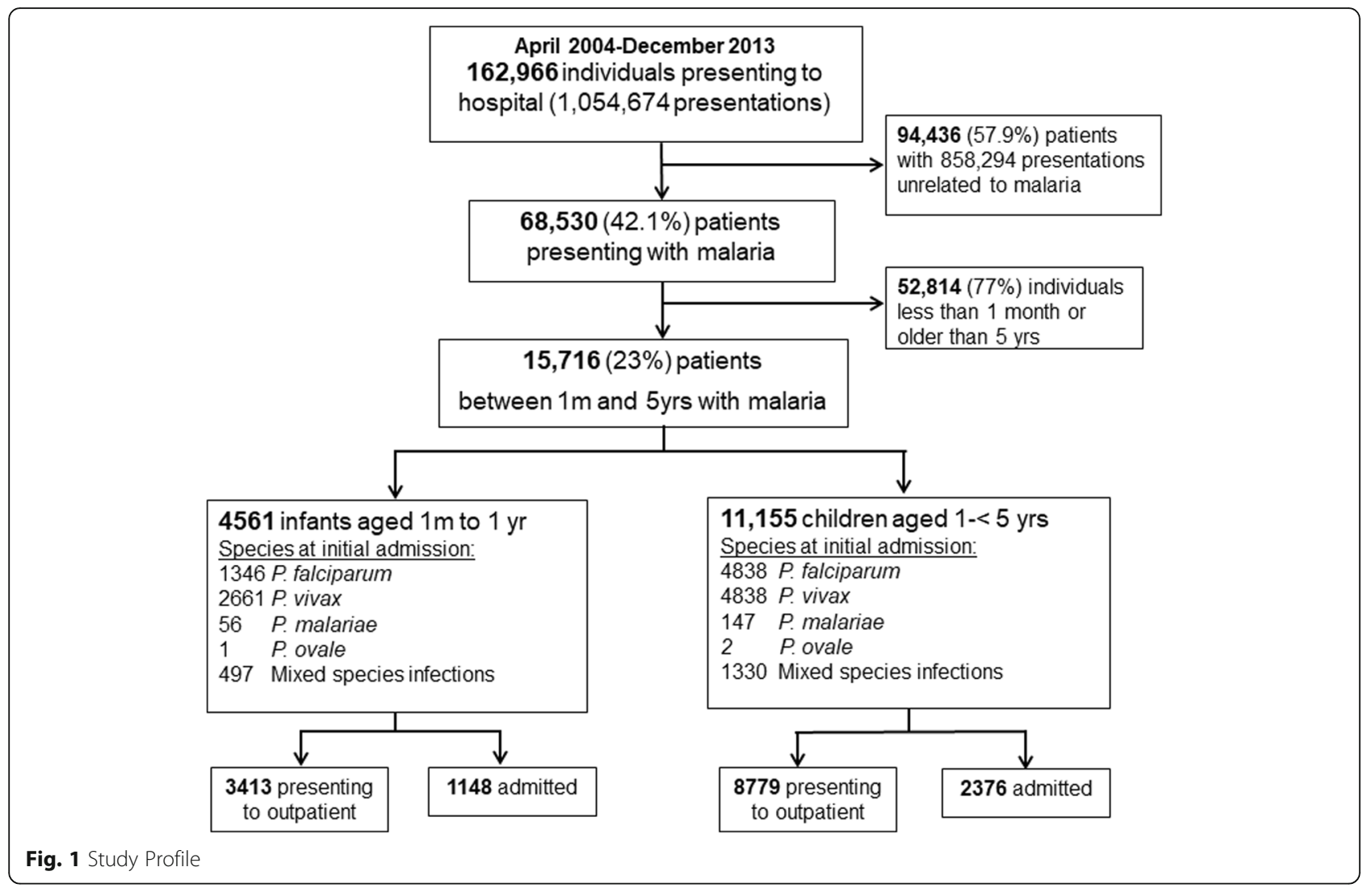

At 12 months, the cumulative risk of any recurrent malaria was 58.9\% [95\%CI 57.4-60.3] in infants compared to 46.7\% [95\% CI 45.8-47.7] in young children; Hazard Ratio $(\mathrm{HR})=1.41$ [95\% CI: 1.34-1.47], $p<0.001$ (Table 2).

Children initially infected with $P$. vivax were at greater risk of recurrent malaria compared to those initially infected with $P$. falciparum ( $\mathrm{HR}=1.48$ [95\%CI: $1.41-1.55]$ ), $p<0.001$ ); Figs. $2 \mathrm{~b}, 3$ and Table 3. Other univariable risk factors for re-presentation with malaria were highland ethnicity (HR $=3.70$ [95\% CI: 3.23-4.24]; $p<0.001)$, admission to hospital ( $\mathrm{HR}=0.73$ [95\%CI: 0.69-0.77]; $p<0.001)$, severe anaemia ( $\mathrm{HR}=0.89$ [95\%CI: 0.83-0.95]; $p<0.001)$ and malnutrition ( $\mathrm{HR}=0.64$ [95\% CI: 0.51-0.82], $p<0.001)$. In multivariable analysis, age, ethnicity, outpatient status, species of initial infection absence of malnutrition and absence of severe anaemia remained significant risk factors for representation with malaria; Table 3.

\section{Mortality}

Of the 15,716 children presenting to the hospital, 266 children died within 12 months with a cumulative risk of 1.7\% (95\%CI: 1.5-1.9). Of these 266 deaths, 94 (35.3\%) occurred within the first 7 days of the initial presentation, 35 (13.2\%) between days 8 to 30, and 137 (51.5\%) between days 31 and 365 (Fig. 4).

\section{Early mortality}

The risk of early mortality was non-significantly higher in infants (1.0\% [95\%CI: 0.8-1.3]) compared to young children (0.7\% [95\%CI: 0.6-0.9]; $p=0.095)$ and those presenting with $P$. falciparum infection $(1.0 \%$ [95\%CI, 0.7-1.2]) compared $P$. vivax infection (0.7\% [95\%CI, 0.6-1.0]; $p=0.156)$.

Early mortality was significantly more likely in undernourished children ( $\mathrm{HR}=19.65$ [95\%CI: 12.86-30.04]); $p<0.001)$, children initially requiring hospitalization $(\mathrm{HR}=10.18$ [95\%CI, 6.85-15.12], $p<0.001)$ and those presenting with severe anaemia $(\mathrm{HR}=2.03$ [95\%CI: $1.41-$ 2.94], $p<0.001$ ); Table 4. Compared to non-Papuans, highland ethnicity was associated with a lower risk of mortality $(\mathrm{HR}=0.46$ [95\%CI $0.27-0.77], p=0.003)$. In multivariable analysis, ethnicity, inpatient status, malnutrition and presenting with severe anaemia remained significant risk factors for early mortality; Table 4. Among undernourished children with malaria, the risk of dying within 30 days was $10.5 \%$ (9/86) in children infected with $P$. falciparum and $15.5 \%(15 / 97)$ in those infected with $P$. vivax; $p=0.437$. In the 2664 (17.0\%) children initially requiring admission to hospital and intravenous treatment with either quinine or artesunate, 3.4\% (28/835) of those with $P$. vivax died within 30 days compared to $1.7 \%$ (24/ $1375)$ of those presenting with $P$. falciparum $(\mathrm{HR}=1.93$ [95\%CI 1.12-3.34]; $p=0.018$ ). 
Table 1 Baseline characteristics of children under 5 years old at their first presentation to hospital with malaria

\begin{tabular}{|c|c|c|c|}
\hline Characteristics & $\begin{array}{l}\text { Total } \\
n=15,716\end{array}$ & $\begin{array}{l}\text { Inpatients } \\
n=3524\end{array}$ & $\begin{array}{l}\text { Outpatients } \\
n=12,192\end{array}$ \\
\hline \multicolumn{4}{|l|}{ Age, years (\%) } \\
\hline Median [Range] & $1.7[0.1,5.0]$ & $1.5[0.1,5.0]$ & $1.8[0.1,5.0]$ \\
\hline $1-5$ years & $11,155(71.0)$ & $2376(67.4)$ & $8779(72.0)$ \\
\hline$<1$ year & $4561(29.0)$ & $1148(32.6)$ & $3413(28.0)$ \\
\hline \multicolumn{4}{|l|}{ Sex, $n(\%)$} \\
\hline Females & $7462(47.5)$ & $1656(47.0)$ & $5806(47.6)$ \\
\hline Male & $8254(52.5)$ & $1868(53.0)$ & $6386(52.4)$ \\
\hline \multicolumn{4}{|l|}{ Ethnic group ${ }^{a}, n(\%)$} \\
\hline Non Papuan & $1100(7.0)$ & $324(9.2)$ & $776(6.4)$ \\
\hline Highland Papuan & $11,714(74.5)$ & $2516(71.4)$ & $9198(75.4)$ \\
\hline Lowland Papuan & $2889(18.4)$ & $679(19.3)$ & $2210(18.1)$ \\
\hline Haemoglobin concentration, $n(\%)$ & $n=8687(55.3 \%)$ & $n=3201(90.8 \%)$ & $n=5486(45.0 \%)$ \\
\hline Mean Haemoglobin [SD] g/dl & $8.1[2.4]$ & $7.3[2.8]$ & $8.6[2.0]$ \\
\hline $\mathrm{Hb}<7 \mathrm{~g} / \mathrm{dl}$ & $2674(30.8)$ & $1441(40.9)$ & $1233(10.1)$ \\
\hline $\mathrm{Hb}<5 \mathrm{~g} / \mathrm{dl}$ & $974(11.2)$ & $814(23.1)$ & $160(1.3)$ \\
\hline \multicolumn{4}{|l|}{ Malnourished, $n(\%)$} \\
\hline Normal & $15,497(98.6)$ & $3368(95.6)$ & $12,129(99.5)$ \\
\hline Undernourished & $219(1.4)$ & $156(4.4)$ & $63(0.5)$ \\
\hline \multicolumn{4}{|l|}{ Species of malaria, $n(\%)$} \\
\hline P. falciparum & $6184(39.3)$ & $1818(51.6)$ & $4366(35.8)$ \\
\hline P. vivax & $7499(47.7)$ & $1146(32.5)$ & $6353(52.1)$ \\
\hline P. ovale & $3(0.02)$ & $0(0.0)$ & $3(0.0)$ \\
\hline P. malariae & $203(1.3)$ & $25(0.7)$ & $178(1.5)$ \\
\hline Mixed Infections & $1827(11.6)$ & $535(15.2)$ & $1292(10.6)$ \\
\hline
\end{tabular}

SD standard deviation; ${ }^{a}$ Data missing for 13 children
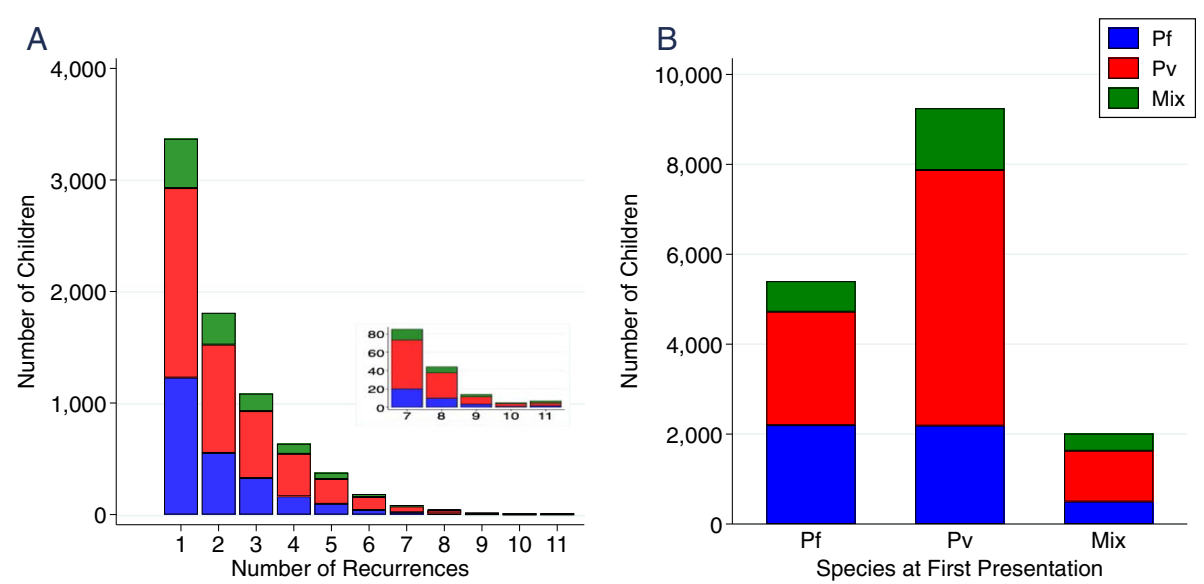

Fig. 2 (a) Histogram showing the distribution of the number of recurrences of malaria stratified by Plasmodium species at recurrence - the first presentation is excluded (b) Total number of recurrences stratified by Plasmodium species at initial presentation and at recurrence 
Table 2 Malaria Incidence Rate within 1 year of initial presentation (per 1000 patient years (95\% CI))

\begin{tabular}{|c|c|c|c|c|}
\hline \multirow[t]{2}{*}{ Outcome 12 months follow-up } & \multicolumn{4}{|c|}{ Species at initial presentation } \\
\hline & Any Malaria & P. falciparum & P. vivax & Mixed Infection \\
\hline Any malaria & $1146(1129,1164)$ & $920(896,944)$ & $1334(1307,1361)$ & $1207(1155,1260$ \\
\hline P. falciparum & $334(325,343)$ & $370(355,386)$ & $314(301,327)$ & $297(272,324)$ \\
\hline P. vivax & $637(624,650)$ & $425(409,442)$ & $815(794,837)$ & $673(635,713)$ \\
\hline Mixed Infections & $166(160,173)$ & $115(106,123)$ & $197(187,208)$ & $227(206,251)$ \\
\hline Inpatient admission with malaria & $411(401,422)$ & $361(346,376)$ & $457(441,473)$ & $415(385,447)$ \\
\hline Outpatient presentation with malaria & $3386(3356,3416)$ & $2875(2832,2919)$ & $3859(3813,3906)$ & $3289(3203,3377$ \\
\hline
\end{tabular}

\section{Late mortality}

In total 137 (0.9\%) children died between 31 and 365 days of their initial presentation. The risk of late mortality was $1.0 \%$ [95\%CI $0.8-1.3]$ in children initially presenting with P. vivax, $0.8 \%$ [95\%CI $0.6-1.0]$ in those initially presenting with $P$. falciparum and $1.0 \%$ [95\%CI 0.6-1.6] in those with mixed infections; $p=0.34$. The risk of late mortality was greater in infants than older children $(\mathrm{HR}=3.11$ [95\%CI: 2.22-4.35], $p<0.001$ ), children requiring admission to hospital compared to those initially managed as an outpatient $(\mathrm{HR}=1.83$ [95\%CI: 1.28-2.60], $p=0.004)$, children who were undernourished $(\mathrm{HR}=2.99$ [95\%CI: $1.22-7.3]$, $p=0.016)$ and those who were severely anaemic $(\mathrm{HR}=$ 1.83 [95\%CI: 1.21-2.79]; $p=0.004)$. Among undernourished children with malaria, the risk of late mortality was $1.2 \%(1 / 82)$ in those initially infected with $P$. falciparum and $3.9 \%(3 / 77)$ in those with $P$. vivax. In total $8 \%(1264 /$ 15716) of children had a recurrent episode of malaria within 30 days, of which $36.3 \%$ (459/1264) were attributable to $P$. falciparum, $51.1 \%(646 / 1264)$ due to $P$. vivax and $12.7 \%(161 / 1264)$ due to mixed infections. Children with any early recurrence of malaria had an increased risk of late mortality ( $\mathrm{HR}=1.94$ [95\%CI: $1.21-3.12]$; $p=0.006)$, which was significant following a $P$. vivax recurrence $(\mathrm{HR}=2.33$ [1.28-4.22]; $p=0.005)$ but not following $P$. fal ciparum recurrence $(\mathrm{HR}=1.34 \quad[0.55-3.28] ; p=0.521)$; Fig. 5, Table 5.

In the 12,065 children initially managed as outpatients, the only independent predictors of late mortality were being an infant $(\mathrm{HR}=3.44$ [95\%CI: $2.27-5.22] ; p<0.001)$ and malnutrition ( $\mathrm{HR}=4.64$ [95\%CI: 1.14-18.85]; $p=0.032)$. Whereas in the 3417 children initially admitted for the management of their malaria, independent predictors of late mortality were being an infant $(\mathrm{HR}=2.38$ [95\% $\mathrm{CI}$ : 1.34-4.21]; $p=0.003$ ) and having another episode of malaria within 30 days $(\mathrm{HR}=3.44$ [95\%CI: $1.66-7.11$; $p<0.001)$.

The overall incidence of malaria was 2280 [95\%CI: 1946-2671] per 1000 py in those who died, compared to 1141 [95\%CI: 1124-1158] for those who didn't die, and similar incidence rates were seen for each initial species at presentation; Table 6.

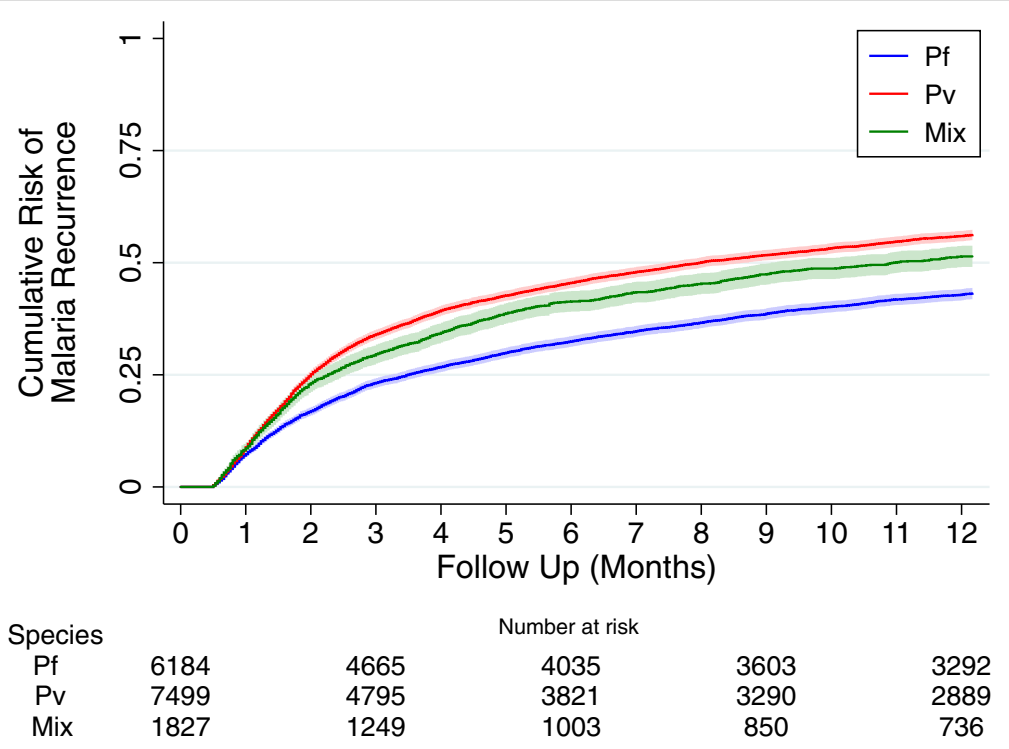

Fig. 3 Risk of representation with malaria within 1 year after initial presentation. Figure stratified by the species at first presentation 
Table 3 Risk factors for malaria re-presentation to hospital within 1 year of initial infection

\begin{tabular}{|c|c|c|c|c|c|c|c|c|c|}
\hline \multirow[b]{2}{*}{ Characteristics } & \multirow[b]{2}{*}{$\begin{array}{l}\text { Risk (num. of } \\
\text { events/total cases) }\end{array}$} & \multirow[b]{2}{*}{$\begin{array}{l}\text { Univariable Analysis } \\
\text { Hazard ratio }(95 \% \mathrm{Cl})\end{array}$} & \multirow[b]{2}{*}{$p$ value } & \multicolumn{3}{|l|}{$\begin{array}{l}\text { Anaemia included in } \\
\text { the model }(n=8680)\end{array}$} & \multicolumn{3}{|c|}{$\begin{array}{l}\text { Anaemia excluded from } \\
\text { the model }(n=15,703)\end{array}$} \\
\hline & & & & $\begin{array}{l}\text { Multivariable Analysis } \\
\text { Adjusted Hazard } \\
\text { Ratio }(95 \% \mathrm{Cl})\end{array}$ & $p$ value & $\begin{array}{l}\text { PAF \% } \\
(95 \% \text { Cl) }\end{array}$ & $\begin{array}{l}\text { Multivariable } \\
\text { Analysis } \\
\text { Adjusted Hazard } \\
\text { Ratio }(95 \% \mathrm{Cl}) \\
\end{array}$ & $\begin{array}{l}p \\
\text { value }\end{array}$ & $\begin{array}{l}\text { PAF \% } \\
(95 \% \mathrm{Cl})\end{array}$ \\
\hline \multicolumn{10}{|l|}{ Age Group } \\
\hline$>=1$ to $<=5$ & $45.2(5044 / 11155)$ & Reference & & Reference & & & Reference & & \\
\hline$<1$ & $56.5(2576 / 4561)$ & $1.41(1.34,1.47)$ & $<0.001$ & $1.35(1.26,1.44)$ & $<0.001$ & $\begin{array}{l}10.34 \\
(7.97 \\
12.66)\end{array}$ & $1.32(1.26,1.38)$ & $\begin{array}{l}< \\
0.001\end{array}$ & $\begin{array}{l}8.84 \\
(7.22 \\
10.44)\end{array}$ \\
\hline \multicolumn{10}{|l|}{ Gender } \\
\hline Female & $48.1(3587 / 7462)$ & Reference & & Reference & & & Reference & & \\
\hline Male & $48.9(4033 / 8254)$ & $1.01(0.97,1.06)$ & 0.562 & $1.04(0.97,1.10)$ & 0.266 & $\begin{array}{l}1.89 \\
(-1.49 \\
5.15)\end{array}$ & $1.02(0.98,1.07)$ & 0.39 & $\begin{array}{l}1.04 \\
(-1.35 \\
3.37)\end{array}$ \\
\hline \multicolumn{10}{|l|}{ Ethnicity $^{a}$} \\
\hline Non Papuan & $19.4(213 / 1100)$ & Reference & & Reference & & & Reference & & \\
\hline Highland & $55.2(6466 / 11714)$ & $3.70(3.23,4.24)$ & $<0.001$ & $3.90(3.21,4.75)$ & $<0.001$ & $\begin{array}{l}65.34 \\
(60.35 \\
69.71)\end{array}$ & $3.48(3.03,3.99)$ & $\begin{array}{l}< \\
0.001\end{array}$ & $\begin{array}{l}62.53 \\
(58.68 \\
66.02)\end{array}$ \\
\hline Lowland & $32.5(939 / 2889)$ & $1.75(1.51,2.03)$ & $<0.001$ & $1.90(1.54,2.35)$ & $<0.001$ & $\begin{array}{l}5.05 \\
(3.66, \\
6.41)\end{array}$ & $1.71(1.48,1.99)$ & $\begin{array}{l}< \\
0.001\end{array}$ & $\begin{array}{l}4.25 \\
(3.22 \\
5.26)\end{array}$ \\
\hline \multicolumn{10}{|l|}{ Inpatient Status } \\
\hline Outpatient & $50.9(6207 / 12192)$ & Reference & & Reference & & & Reference & & \\
\hline Inpatient & $40.1(1413 / 3524)$ & $0.73(0.69,0.77)$ & $<0.001$ & $0.82(0.76,0.88)$ & $<0.001$ & $\begin{array}{l}-6.68 \\
(-9.01,- \\
4.40)\end{array}$ & $0.78(0.74,0.83)$ & $\begin{array}{l}< \\
0.001\end{array}$ & $\begin{array}{l}-4.85 \\
(-5.97,- \\
3.75)\end{array}$ \\
\hline \multicolumn{10}{|l|}{ Anaemiał } \\
\hline $\mathrm{Hb}>=7 \mathrm{~g} / \mathrm{dl}$ & $48.3(2904 / 6013)$ & Reference & & Reference & & & - & - & - \\
\hline $\mathrm{Hb}<7 \mathrm{~g} / \mathrm{dl}$ & $44.2(1182 / 2674)$ & $0.89(0.83,0.95)$ & $<0.001$ & $0.89(0.83,0.96)$ & 0.002 & $\begin{array}{l}-3.29 \\
(-5.40,- \\
1.23)\end{array}$ & - & - & - \\
\hline \multicolumn{10}{|l|}{ Malnourished } \\
\hline Normal & $48.7(7552 / 15497)$ & Reference & & Reference & & & Reference & & \\
\hline Undernourished & $31.1(68 / 219)$ & $0.64(0.51,0.82)$ & $<0.001$ & $0.75(0.58,0.97)$ & 0.026 & $\begin{array}{l}-0.51 \\
(-0.90,- \\
0.12)\end{array}$ & $0.75(0.59,0.96)$ & 0.021 & $\begin{array}{l}-0.29 \\
(-0.50,- \\
0.07)\end{array}$ \\
\hline \multicolumn{10}{|c|}{ Species at presentation } \\
\hline P. falciparum & $42.0(2597 / 6184)$ & Reference & & Reference & & & Reference & & \\
\hline P. vivax & $54.0(4048 / 7499)$ & $1.48(1.41,1.55)$ & $<0.001$ & $1.24(1.16,1.33)$ & $<0.001$ & $\begin{array}{l}10.14 \\
(6.82 \\
13.34)\end{array}$ & $1.31(1.24,1.37)$ & $\begin{array}{l}< \\
0.001\end{array}$ & $\begin{array}{l}13.11 \\
(10.61 \\
15.53)\end{array}$ \\
\hline P. ovale & $66.7(2 / 3)$ & $1.99(0.50,7.97)$ & 0.33 & $3.58(0.89,14.40)$ & 0.073 & $\begin{array}{l}0.06 \\
(-0.06 \\
0.18)\end{array}$ & $2.54(0.63,10.18)$ & 0.188 & $\begin{array}{l}0.02(- \\
0.03 \\
0.08)\end{array}$ \\
\hline P. malariae & $37.4(76 / 203)$ & $0.85(0.68,1.07)$ & 0.163 & $0.75(0.54,1.05)$ & 0.096 & $\begin{array}{l}-0.25(- \\
0.51 \\
0.01)\end{array}$ & $0.82(0.65,1.03)$ & 0.088 & $\begin{array}{l}-0.19 \\
(-0.40 \\
0.01)\end{array}$ \\
\hline Mix & $49.1(897 / 1827)$ & $1.30(1.20,1.40)$ & $<0.001$ & $1.20(1.09,1.33)$ & $<0.001$ & $\begin{array}{l}2.37 \\
(1.02, \\
3.69)\end{array}$ & $1.19(1.10,1.29)$ & $\begin{array}{l}< \\
0.001\end{array}$ & $\begin{array}{l}1.92 \\
(1.02,2.8)\end{array}$ \\
\hline
\end{tabular}

All multivariable models were stratified by year. In view of the change in antimalarial treatment policy in 2006 and collinearity of year and treatment, oral and intravenous therapy received was not entered in the multivariable models. PAF population attributable fraction. ${ }^{\text {a }}$ Data missing for 13 children 


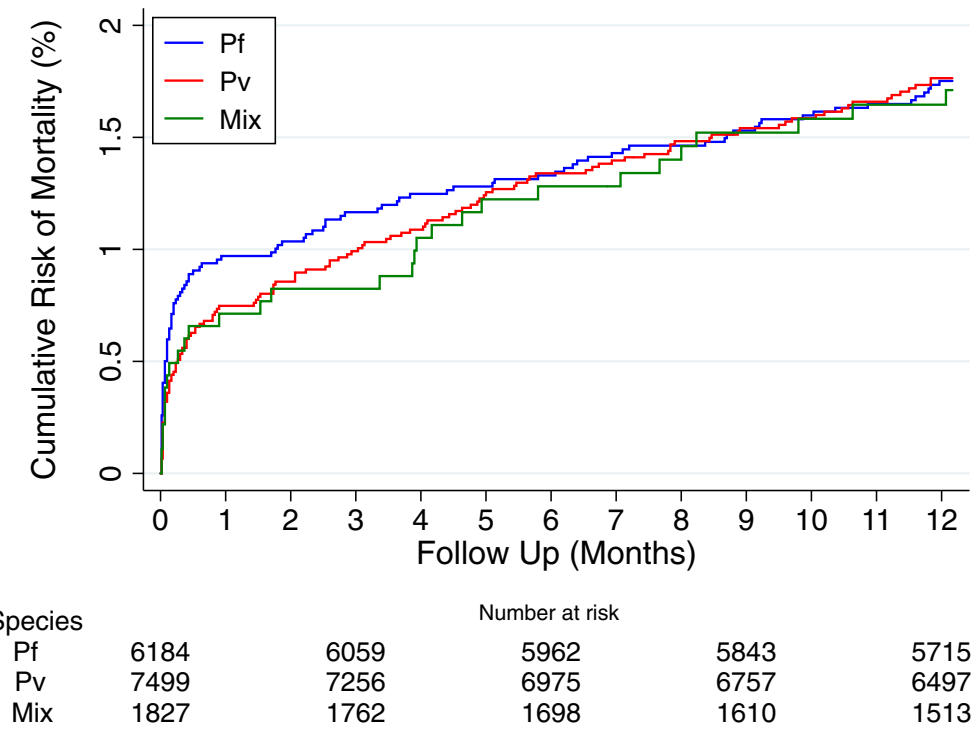

Fig. 4 Risk of mortality within 1 year after initial presentation. Figure stratified by the species at first presentation

\section{Diagnosis at the time of death}

Of the 129 children who died within 30 days of their initial presentation with malaria, $21.7 \%$ (28/129) were malnourished, with pneumonia reported in $28.7 \%(37 / 129)$ and diarrhea in $19.4 \%(25 / 129)$. Of those 112 children with documented haemoglobin concentrations, 55 (49.1\%) were severely anaemic. Of the 137 children surviving their initial episode of malaria but subsequently dying between 31 days and 1 year, $37(27.0 \%)$ had recurrent malaria at the time of death, 47 (34.3\%) were malnourished, 42 (30.7\%) had pneumonia and 40 (29.2\%) had diarrhea. Of the 113 children with documented haemoglobin concentrations, $47(41.6 \%)$ were severely anaemic at the time of death. There was no significant difference in the risk of these comorbidities between those presenting with $P$. falciparum and those with $P$. vixax.

\section{Discussion}

The acute morbidity and mortality of $P$. vivax malaria has been documented in a range of endemic settings $[3,4,8$, 22], however the delayed consequences, particularly those associated with recurrent parasitaemia, have not been defined. In the current study the acute and delayed detrimental effects of $P$. vivax on health and survival were assessed in infants and young children in Papua Indonesia, an area co-endemic for $P$. vivax and P. falciparum.

At the acute presentation, children with $P$. falciparum infection were 2.3 -fold more likely to be admitted to hospital for inpatient care. The 30-day risk of mortality was higher for children with $P$. falciparum than those with $P$. vivax (1.0\% vs $0.7 \%)$, although this did not reach statistical significance. Conversely, the delayed risk of dying (day 31 to 365) was higher in children initially presenting with $P$. vivax (1.0\% vs $0.8 \%$ ), and by 12 months the cumulative risk of mortality was almost identical between the two species; Fig. 4 .

We were unable to identify children fulfilling World Health Organization criteria for severe malaria since the necessary clinical and laboratory criteria were not recorded in our surveillance data (with the exception of haemoglobin). However pharmacy data were documented and allowed us to identify those who were treated with intravenous antimalarial drugs (quinine or artesunate). Intravenous artesunate was introduced for severe malaria due to any species in March 2006, and clinicians have a low threshold for prescribing this in those with clinical concern. Interestingly in patients receiving intravenous therapy, those with $P$. vivax were almost 2-fold more likely to die during their hospital admission compared to those with $P$. falciparum and this difference remained after controlling for confounding factors. Intravenous artesunate is hypothesized to reduce the mortality of $P$. falciparum infection by its rapid reduction in the parasite biomass and prevention of microvascular sequestration and end organ dysfunction [17]. Severe disease in vivax malaria has a lower parasite biomass than that observed in falciparum malaria [23] and its pathophysiology is characterised by severe anaemia, acute inflammation and microvascular dysfunction associated with comorbidities such as malnutrition, pneumonia and diarrhea $[7,8]$. In these circumstances a rapid reduction of parasite biomass may have less impact on the clinical outcome.

In the current study, almost half of the children treated for malaria re-presented within 12 months with a further episode of malaria, some children experiencing up to 11 recurrences. The overall incidence of malaria 


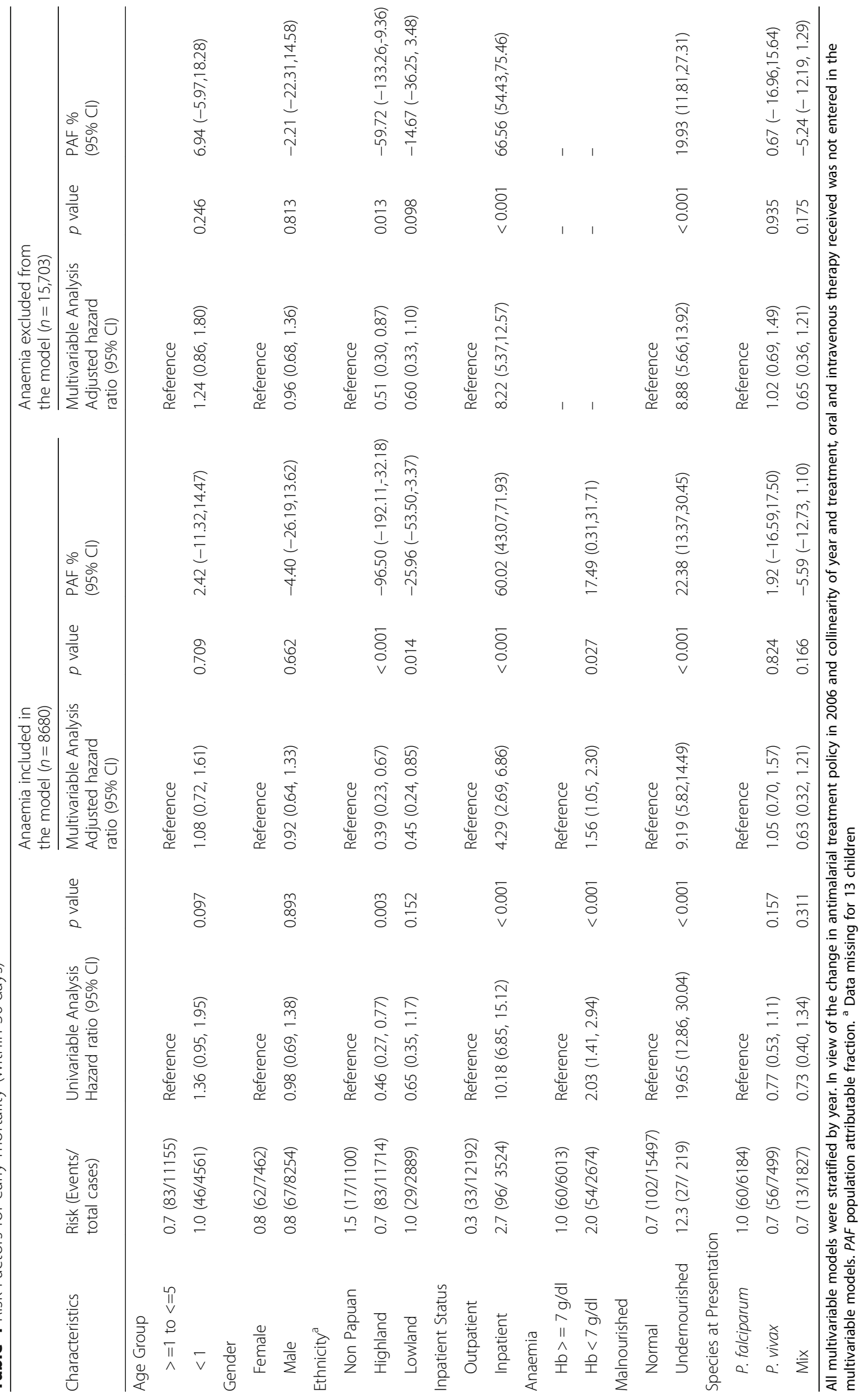



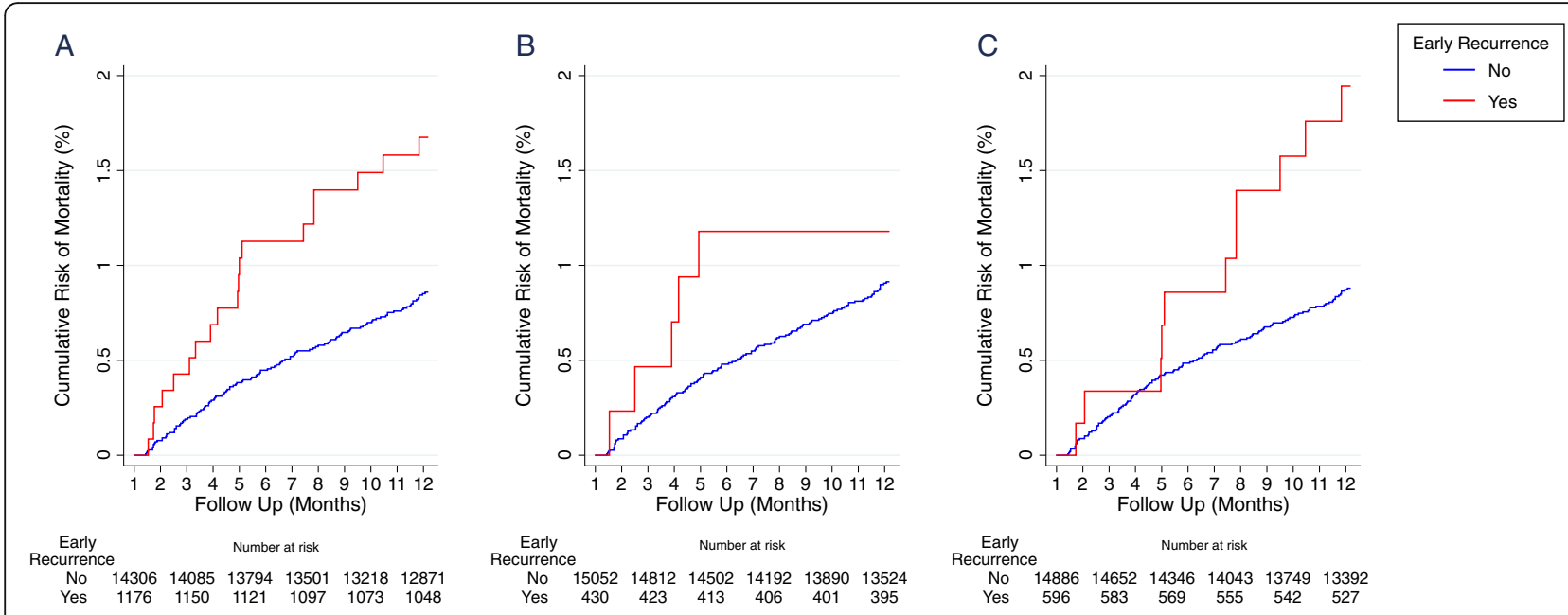

Fig. 5 Risk of mortality stratified on the recurrence of malaria within 30 days of first presentation. a any parasitaemia, $\mathbf{b} P$. falciparum parasitaemia and $\mathbf{c}$ P. vivax parasitaemia. Footnote: Red: parasitaemia within 30 days; Blue: no parasitaemia within 30 days

after an initial episode was 1146 per 1000 py. The ability of $P$. vivax to form dormant liver stages, results in the parasite relapsing weeks to months following the primary infection and this was reflected by those presenting with $P$. vivax being at significantly greater risk of any recurrence and multiple recurrences compared to those presenting with $P$. falciparum.

Early parasite recurrence following discharge increased the risk of late mortality by almost 2-fold, and this effect was apparent throughout the 12 months of follow-up (Fig. 5), suggesting that early parasite recurrence may exacerbate or reflect the presence of other risk factors for death. Patients with early recurrent parasitaemia may also be at greater risk for subsequent malaria infections. Indeed, those patients who died within a year had a 2-fold higher incidence of malaria compared to those who survived.

Pro-inflammatory cytokines associated with acute parasitaemia can induce anorexia and increase catabolism, and this is likely to contribute to malnutrition which has been associated with recurrent malaria [2426]. In the Pacific, children with $P$. vivax infections have been shown to be at higher risk of malnutrition compared to those with P. falciparum infections [27].

The chronic and relapsing nature of $P$. vivax results in repeated episodes of haemolysis and dyserythropoeisis $[6,28]$. When parasite recurrence occurs prior to haematological recovery, there is a cumulative risk of severe anaemia which, when coupled with malnutrition and sepsis, can result in a fatal outcome [7, 29]. In the current analysis, severe anaemia $(\mathrm{Hb}<7 \mathrm{~g} / \mathrm{dl})$ was associated with a 2-fold increased risk of delayed mortality and malnutrition with a 3-fold increased risk.

The under-five mortality rate in at the RSMM hospital, during the study period, was 61 per 1000 live births, with $72 \%$ of deaths occurring within the first 7 days of life (unpublished data). Hence in babies surviving the neonatal period, the overall risk of dying was approximately $0.3 \%$ per year. In the current analysis the corresponding risk of mortality in young children presenting with $P$. vivax malaria was conservatively estimated at $1 \%$, three fold higher than expected. Our study suggests that recurrent episodes of malaria may have contributed both directly and indirectly to this high mortality rate. At the time of death, only $42 \%$ of children were associated with another episode of malaria, whereas, $28 \%$ were malnourished, $45.3 \%$ had severe anaemia and 55\% presented with infective comorbidities such as pneumonia or diarrhea. Whilst we are unable to discern the proportion of deaths directly attributable to recurrent episodes of malaria, our findings suggest that the management of acute malaria must include both acute treatment of peripheral parasitaemia, malnutrition and severe anaemia, as well as interventions to prevent subsequent malaria recurrence, such as insecticide treated bednets, prophylaxis or radical cure. The latter requires administration of a combination of a blood schizontocidal drug with a hypnozoitocidal agent. The only currently available drug that targets the dormant liver stage is primaquine, an 8-aminoquinoline compound, which can cause severe heamolysis in G6PD deficient individuals. Concerns over its potential toxicity have limited its use in young children. The World Health Organization recommends that in areas where G6PD testing is unavailable, the use of primaquine should be based on careful assessment of risk and benefit of the treatment [30]. Our findings highlight the significant risks of not providing effective antirelapse therapy.

This study has several important limitations. Firstly, the medical records collected included limited clinical data and relied upon the diagnosis made by the attending physician, coded according to the International 
Table 5 Risk Factors for late mortality (between 31 and 365 days)

\begin{tabular}{|c|c|c|c|}
\hline Characteristics & Risk (Events/total cases) & $\begin{array}{l}\text { Univariable Analysis } \\
\text { Hazard ratio }(95 \% \mathrm{Cl})\end{array}$ & $p$ value \\
\hline \multicolumn{4}{|l|}{ Age Group } \\
\hline$>=1$ to $<=5$ & $0.6(61 / 11005)$ & Reference & \\
\hline$<1$ & $1.7(76 / 4477)$ & $3.11(2.22,4.35)$ & $<0.001$ \\
\hline \multicolumn{4}{|l|}{ Gender } \\
\hline Female & $1.0(70 / 7346)$ & Reference & \\
\hline Male & $0.8(67 / 8136)$ & $0.86(0.62,1.20)$ & 0.38 \\
\hline \multicolumn{4}{|l|}{ Ethnicity $^{a}$} \\
\hline Non Papuan & $0.2(2 / 1073)$ & Reference & \\
\hline Highland & $1.1(131 / 11545)$ & $6.08(1.50,24.55)$ & 0.011 \\
\hline Lowland & $0.1(4 / 2851)$ & $0.74(0.14,4.04)$ & 0.728 \\
\hline \multicolumn{4}{|l|}{ Inpatient Status } \\
\hline Outpatient & $0.7(90 / 12065)$ & Reference & \\
\hline Inpatient & $1.4(47 / 3417)$ & $1.83(1.28,2.60)$ & $<0.001$ \\
\hline \multicolumn{4}{|l|}{ Anaemia $^{\mathrm{b}}$} \\
\hline $\mathrm{Hb}>=7 \mathrm{~g} / \mathrm{dl}$ & $0.8(49 / 5918)$ & Reference & \\
\hline $\mathrm{Hb}<7 \mathrm{~g} / \mathrm{dl}$ & $1.5(40 / 2612)$ & $1.83(1.21,2.79)$ & 0.004 \\
\hline \multicolumn{4}{|l|}{ Malnourished } \\
\hline Normal & $0.9(132 / 15290)$ & Reference & \\
\hline Undernourished & $2.6(5 / 192)$ & $2.99(1.22,7.30)$ & 0.016 \\
\hline \multicolumn{4}{|c|}{ Recurrent malaria within 30 days } \\
\hline None & $0.8(117 / 14234)$ & Reference & \\
\hline P. falciparum & $1.1(5 / 452)$ & $1.34(0.55,3.28)$ & 0.521 \\
\hline P. vivax & $1.9(12 / 635)$ & $2.33(1.28,4.22)$ & 0.005 \\
\hline Mixed Infections & $1.9(3 / 161)$ & $2.34(0.74,7.37)$ & 0.145 \\
\hline \multicolumn{4}{|c|}{ Species at presentation } \\
\hline P. falciparum & $0.8(47 / 6106)$ & Reference & \\
\hline P. vivax & $1.0(72 / 7378)$ & $1.30(0.90,1.88)$ & 0.157 \\
\hline Mixed Infections & $0.9(17 / 1793)$ & $1.28(0.73,2.23)$ & 0.386 \\
\hline
\end{tabular}

a Data missing for 13 children; ${ }^{\mathrm{b}}$ Data on haemoglobin concentrations missing in 6952 children. The low number of late deaths in some subgroups, precluded the presentation of a reliable multivariable model

Table 6 Incidence rate of malaria and re-presentation to hospital in those dying and surviving in subsequent 12 months

\begin{tabular}{lll}
\hline Recurrence Outcome & Died & Alive \\
\hline Any malaria & $2280(1946,2671)$ & $1141(1124,1158)$ \\
P. falciparum & $596(437,813)$ & $333(323,342)$ \\
P. vivax & $1401(1144,1715)$ & $634(621,647)$ \\
Mixed Infections & $238(146,389)$ & $166(159,173)$ \\
Inpatient admission & $3249(2845,3710)$ & $396(386,406)$ \\
Outpatient presentations & $5830(5280,6438)$ & $3376(3347,3406)$ \\
\hline
\end{tabular}

Figures are per 1000 patient years $(95 \% \mathrm{Cl})$

Excludes 129 patients who died within the first 30 days
Classifications of the Disease 10 (ICD-10). The diagnosis of severe malaria was not captured, however, patients with severe malaria would be more likely to be admitted and be treated with intravenous antimalarials. We assumed that all deaths from severe malaria would have occurred within 31 days. In a previous study at the same location, we showed the important contribution of sepsis and comorbidities to malaria attributable mortality [7]. Since our current analysis did not quantify the effects of recurrent episodes of non-malarial illness to mortality, we cannot infer the fraction of late mortality attributable to recurrent malaria compared with other comorbidities. However we speculate that recurrent episodes of malaria result in a cumulative risk of anaemia and malnutrition that increases the vulnerability of infants to severe manifestations of other concomitant infections common in early childhood. 
In support of this, the incidence of malaria was much greater in children who died than those who survived (Table 6), yet when death occurred after 30 days only $27 \%$ of cases were associated with recurrent malaria.

This study was limited to patients attending RSMM for healthcare who were mainly from one of the 7 Papuan ethnic groups, are entitled to free health services. Since we did not gather data from patients who represented at other healthcare facilities, a proportion of patients who died in the community or visited other health facilities for malaria treatment would have been missed. The primary aim of this analysis was to determine the comparative risks of recurrent malaria and death between patients initially presenting with $P$. falciparum versus those presenting with $P$. vivax. Previous community surveys have shown that patients with malaria due to either $P$. falciparum or $P$. vivax have a similar tendency to seek medical attention at the same location and in this region, a high proportion of deaths in young children occur at RSMM hospital $[12,13]$. We therefore assumed that the attrition bias in detecting the clinical endpoints of the study were similar between species. Hence our comparative analysis remains valid and indeed our findings represent conservative estimates of the risk of recurrence and early and late mortality. In view of the co-linearity between the year of observation and malaria treatment protocol, this study could not compare the effect of treatment on mortality. Hence our analysis represents comparative mortality for the current standard of care at presentation.

Lastly, our estimates of the comparative crude incidence of malaria in those who died versus those who survived are almost certainly biased by the development of immunity and also by differences in age. Children who die are most likely to do so before the development of effective immunity and therefore will have a greater chance of clinical malaria episodes leading up to death than those who survived. Those who died were on average younger than those who survived and it is well recognized that very young children have the highest incidence of malaria (particularly due to $P$. vivax) in Timika.

\section{Conclusions}

Children under 5 years old presenting with malaria due to either $P$. vivax and/or $P$. falciparum have a significant risk of representation with malaria, admission to hospital and both acute and delayed mortality. Recurrent malaria, malnutrition and anaemia were associated with an increased risk of delayed mortality. In the context of malaria elimination in Indonesia, our study suggests that focused efforts are warranted to ensure that early detection and prompt, effective treatment aimed at both eradication of the acute infection and prevention of subsequent recurrence should be delivered in this high risk population.

\section{Abbreviations}

ACT: Artemisinin Combination Therapy; Cl: Confidence Interval;

DHP: Dihydroartemisinin piperaquine; G6PD: Glucose-6-phosphate dehydrogenase; HR: Hazard Ratio; ICD-10: International Classifications of the Disease - 10; PQ: Primaquine; py: person-years; RSMM: Rumah Sakit Mitra Masyarakat (Mitra Masyarakat Hospital); SD: Standard deviation

\section{Acknowledgements}

We are grateful to Lembaga Pengembangan Masyarakat Amungme Kamoro and Mitra Masyarakat Hospital (RSMM) in Timika, Papua, Indonesia. We thank Professor Yati Soenarto and Dr. Yodi Mahendradhata from the Faculty of Medicine Universitas Gadjah Mada, Yogyakarta for their excellent support to the study.

\section{Authors' contributions}

$D P, E A, N M D, R N P$ and JRP developed the research questions and methodology. EK and DAL collected the data. PS and AH supervised the data collection and study protocol. DP, JRP, RNP and SD, analysed the data. DP and JRP wrote the first draft of the manuscript. JAS, NMD, NMA and RNP contributed to data interpretation and manuscript revisions. All authors read and approved the final manuscript.

\section{Funding}

The study was funded by the Wellcome Trust through a Senior Fellowship in Clinical Science awarded to RNP (200909) and Training Fellowship in Tropical Medicine awarded to JRP (099875) and Australian National Health and Medical Research Council (NHMRC): Senior Research Fellowship to JAS (1104975) and Senior Principal Research Fellowship to NMA (1135820). The Timika Research Facility and Papuan Health and Community Development Foundation were supported by AusAID (Australian Agency for International Development, Department of Foreign Affairs and Trade) and the National Health and Medical Research Council of Australia (Program Grant 1037304). This work was supported by the Australian Centre for Research Excellence on Malaria Elimination, funded by the NHMRC (1134989). The funding body had no role in the design of the study and collection, analysis and interpretation of data and in writing the manuscript.

\section{Ethics approval and consent to participate}

Ethical approval for this study was obtained from the Medical and Health Research Ethics Committee, Faculty of Medicine, Gadjah Mada University, Yogyakarta, Indonesia (KE/FK/544/EC), Menzies School of Health Research, Darwin, Australia (HREC 10.1397). The data used in this study is a secondary data from hospital electronic records and therefore individual parental consent was not obtained. Patient's identification used in this study is hospital record number without name and address. The process was approved by both the ethics review board of the University of Gadjah Mada in Indonesia and Menzies School of Health in Australia.

Consent for publication

Not applicable.

\section{Competing interests}

The authors declare that they have no competing interests.

\section{Author details}

${ }^{1}$ Department of Child Health, Faculty of Medicine, Public Health and Nursing, Universitas Gadjah Mada, Jl. Kesehatan no.1, Sekip, Yogyakarta 55284, Indonesia. ${ }^{2}$ Timika Malaria Research Programme, Papuan Health and Community Development Foundation, JI. SP2-SP5, RSMM Area, Timika, Papua 99910, Indonesia. ${ }^{3}$ Mimika District Hospital, J. Yos Sudarso, Timika, Papua 99910, Indonesia. ${ }^{4}$ Centre for Epidemiology and Biostatistics, Melbourne School of Population and Global Health, 207 Bouverie Street, The University of Melbourne, Melbourne, Victoria 3010, Australia. ${ }^{5}$ Mitra Masyarakat Hospital, Jl. SP2-SP5-Charitas, Timika 99910, Indonesia. ${ }^{6} \mathrm{Global}$ and Tropical Health Division, Menzies School of Health Research and Charles Darwin University, PO Box 41096, Casuarina, Darwin, NT 0811, Australia. ${ }^{7}$ Division of Medicine, Royal Darwin Hospital, Darwin, NT 0810, Australia. ${ }^{8}$ Centre for Tropical Medicine and Global Health, Nuffield Department of Clinical Medicine, University of Oxford, OX37L, Oxford, United Kingdom. ${ }^{9}$ Mahidol-Oxford Tropical Medicine Research Unit, Faculty of Tropical Medicine, Mahidol University, Bangkok, Thailand. 
Received: 28 April 2018 Accepted: 23 September 2019

Published online: 30 October 2019

\section{References}

1. World Health Organisation. Severe malaria. Trop Med Int Health. 2014; 19(Suppl 1):7-131.

2. Poespoprodjo JR, Fobia W, Kenangalem E, Lampah DA, Hasanuddin A Warikar N, Sugiarto P, Tjitra E, Anstey NM, Price RN. Vivax malaria: a major cause of morbidity in early infancy. Clin Infect Dis. 2009;48(12):1704-12.

3. Tjitra E, Anstey NM, Sugiarto P, Warikar N, Kenangalem E, Karyana M, Lampah DA, Price RN. Multidrug-resistant Plasmodium vivax associated with severe and fatal malaria: a prospective study in Papua, Indonesia. PLoS Med. 2008:5(6):e128.

4. Genton B, D'Acremont V, Rare L, Baea K, Reeder JC, Alpers MP, Muller I. Plasmodium vivax and mixed infections are associated with severe malaria in children: a prospective cohort study from Papua New Guinea. PLoS Med. 2008:5(6):e127.

5. White NJ. Determinants of relapse periodicity in Plasmodium vivax malaria. Malar J. 2011:10:297.

6. Douglas NM, Lampah DA, Kenangalem E, Simpson JA, Poespoprodjo JR, Sugiarto P, Anstey NM, Price RN. Major burden of severe anemia from nonfalciparum malaria species in southern Papua: a hospital-based surveillance study. PLoS Med. 2013;10(12):e1001575.

7. Douglas NM, Pontororing GJ, Lampah DA, Yeo TW, Kenangalem E, Poespoprodjo JR, Ralph AP, Bangs MJ, Sugiarto P, Anstey NM, et al. Mortality attributable to Plasmodium vivax malaria: a clinical audit from Papua, Indonesia. BMC Med. 2014;12:217.

8. Anstey NM, Douglas NM, Poespoprodjo JR, Price RN. Plasmodium vivax: clinical Spectrum, risk factors and pathogenesis. Adv Parasitol. 2012;80:151-201.

9. Kenangalem E, Poespoprodjo JR, Douglas NM, Burdam FH, Gdeumana K, Chalfein F, Prayoga TF, Devine A, Marfurt J, et al. Malaria morbidity and mortality following introduction of a universal policy of artemisinin-based treatment for malaria in Papua, Indonesia: a longitudinal surveillance study. PLoS Med. 2019;16(5):e1002815

10. Sumawinata IW, Bernadeta LB, Sutamihardja A, Purnomo SB, Sekartuti FDJ, Baird JK. Very high risk of therapeutic failure with chloroquine for uncomplicated Plasmodium falciparum and P. vivax malaria in Indonesian Papua. Am J Trop Med Hyg. 2003;68(4):416-20.

11. Ratcliff A, Siswantoro $H$, Kenangalem E, Wuwung M, Brockman A, Edstein MD, Laihad F, Ebsworth EP, Anstey NM, Tjitra E, et al. Therapeutic response of multidrug-resistant Plasmodium falciparum and $P$. vivax to chloroquine and sulfadoxine-pyrimethamine in southern Papua, Indonesia. Trans R Soc Trop Med Hyg. 2007;101(4):351-9.

12. Devine A, Kenangalem E, Burdam FH, Anstey NM, Poespoprodjo JR, Price RN, Yeung S. Treatment-seeking behavior after the implementation of a unified policy of Dihydroartemisinin-Piperaquine for the treatment of uncomplicated malaria in Papua, Indonesia. Am J Trop Med Hyg. 2018;98(2):543-50.

13. Karyana M, Devine A, Kenangalem E, Burdarm L, Poespoprodjo JR, Vemuri R, Anstey NM, Tjitra E, Price RN, Yeung S. Treatment-seeking behaviour and associated costs for malaria in Papua, Indonesia. Malar J. 2016;15(1):536.

14. Poespoprodjo JR, Fobia W, Kenangalem E, Hasanuddin A, Sugiarto P, Tjitra E, Anstey NM, Price RN. Highly effective therapy for maternal malaria associated with a lower risk of vertical transmission. J Infect Dis. 2011;204:1613-9.

15. Poespoprodjo JR, Fobia W, Kenangalem E, Lampah DA, Warikar N, Seal A, McGready R, Sugiarto P, Tjitra E, Anstey NM, et al. Adverse pregnancy outcomes in an area where multidrug-resistant plasmodium vivax and Plasmodium falciparum infections are endemic. Clin Infect Dis. 2008;46(9):1374-81.

16. Ratcliff A, Siswantoro H, Kenangalem E, Maristela R, Wuwung RM, Laihad F, Ebsworth EP, Anstey NM, Tjitra E, Price RN. Two fixed-dose artemisinin combinations for drug-resistant falciparum and vivax malaria in Papua, Indonesia: an open-label randomised comparison. Lancet. 2007;369(9563):757-65.

17. Dondorp A, Nosten F, Stepniewska K, Day N, White N. South east Asian quinine Artesunate malaria trial g: Artesunate versus quinine for treatment of severe falciparum malaria: a randomised trial. Lancet. 2005:366(9487):717-25.

18. CDC Growth Charts [http://www.cdc.gov/growthcharts/background.htm]. Accessed 22 Mar 2017

19. WHO CHild Growth Standards: Methods and Development [http://www who.int/childgrowth/standards/weight_for_age/en/index.html]. Accessed 19 Oct 2010.

20. Douglas NM, Poespoprodjo JR, Patriani D, Malloy MJ, Kenangalem E, Sugiarto P, Simpson JA, Soenarto Y, Anstey NM, Price RN. Unsupervised primaquine for the treatment of Plasmodium vivax malaria relapses in southern Papua: a hospital-based cohort study. PLoS Med. 2017:14(8):e1002379.

21. Newson RB. Attributable and unattributable risks and fractions and other scenario comparisons. Stata J. 2013;13(4):672-98.

22. Kochar DK, Saxena V, Singh N, Kochar SK, Kumar SV, Das A. Plasmodium vivax malaria. Emerg Infect Dis. 2005;11(1):132-4.

23. Barber BE, William T, Grigg MJ, Parameswaran U, Piera KA, Price RN, Yeo TW, Anstey NM. Parasite biomass-related inflammation, endothelial activation, microvascular dysfunction and disease severity in vivax malaria. PLoS Pathog. 2015;11(1):e1004558.

24. Nyakeriga AM, Troye-Blomberg M, Chemtai AK, Marsh K, Williams TN. Malaria and nutritional status in children living on the coast of Kenya. Am J Clin Nutr. 2004;80(6):1604-10.

25. Caulfield LE, Richard SA, Black RE. Undernutrition as an underlying cause of malaria morbidity and mortality in children less than five years old. Am J Trop Med Hyg. 2004;71(2 Suppl):55-63.

26. Tracey KJ, Cerami A. Tumor necrosis factor in the malnutrition (cachexia) of infection and cancer. Am J Trop Med Hyg. 1992;47(1 Pt 2):2-7.

27. Williams TN, Maitland K, Phelps L, Bennett S, Peto TE, Viji J, Timothy R, Clegg JB, Weatherall DJ, Bowden DK. Plasmodium vivax: a cause of malnutrition in young children. QJM. 1997;90(12):751-7.

28. Douglas NM, Anstey NM, Buffet PA, Poespoprodjo JR, Yeo TW, White NJ, Price RN. The anaemia of Plasmodium vivax malaria. Malar J. 2012;11(1):135.

29. Bhattacharya SK, Sur D, Dutta S, Kanungo S, Ochiai RL, Kim DR, Anstey NM, von Seidlein L, Deen J. Vivax malaria and bacteraemia: a prospective study in Kolkata, India. Malar J. 2013;12:176.

30. World Health Organisation. Guidelines for the treatment of malaria. 3rd ed. Geneva: WHO; 2015

\section{Publisher's Note}

Springer Nature remains neutral with regard to jurisdictional claims in published maps and institutional affiliations.
Ready to submit your research? Choose BMC and benefit from:

- fast, convenient online submission

- thorough peer review by experienced researchers in your field

- rapid publication on acceptance

- support for research data, including large and complex data types

- gold Open Access which fosters wider collaboration and increased citations

- maximum visibility for your research: over $100 \mathrm{M}$ website views per year

At BMC, research is always in progress.

Learn more biomedcentral.com/submissions 